\title{
Ultra-wide-angle Wireless Endoscope with a Backend-camera-controller Architecture
}

\author{
Dongha Shim and Jason Yi
}

\begin{abstract}
This paper presents a wireless endoscope with an ultra-wide FOV (Field of View) of $130^{\circ}$ and $\mathrm{HD}$ resolution (1280x720 pixels). The proposed endoscope consists of a camera head, cable, camera controller, and wireless handle. The lens module with a $150^{\circ}$ degrees $\mathrm{AOV}$ (Angle of View) is achieved using the plastic injection-molding process to reduce manufacturing costs. A serial CMOS image sensor using the MIPI (Mobile Industry Processor Interface) CSI-2 (Camera Serial Interface-2) interface physically separates the camera processor from the camera head. The camera head and the cable have a compact structure due to the BCC (Backend-Camera-Controller) architecture. The size of the camera head and the camera controller is $8 \times 8 \times 26 \mathrm{~mm}$ and $7 \times 55 \mathrm{~mm}$, respectively. The wireless handle supports a UWB (Ultra-Wide-Band) or a Wi-Fi communication to transmit video data. The UWB link supports a maximum data transfer rate of $\sim 37 \mathrm{Mbps}$ to transmit video data with a resolution of $1280 \times 720$ pixels at a frame rate of $30 \mathrm{fps}$ in the MJPEG (Motion JPEG) format. Although the Wi-Fi link provides a lower data transfer rate ( $\sim 8 \mathrm{Mbps}$ Max.), it has the advantage of flexible interoperability with various mobile devices. The latency of the UWB link is measured to be $\sim 0.1 \mathrm{sec}$. The Wi-Fi link has a larger latency $(\sim 0.5 \mathrm{sec})$ due to its lower data transfer rate. The proposed endoscope demonstrates the feasibility of a high-performance yet low-cost wireless endoscope using the BCC architecture. To the best of the author's knowledge, the proposed endoscope has the largest FOV among all presently existing wireless endoscopes.
\end{abstract}

Keywords - Wireless endoscope, ultra-wide-angle, plastic lens, backend-camera-controller, serial image sensor, ultra-wide-band, Wi-Fi, latency

\section{INTRODUCTION}

A $\mathrm{N}$ endoscope has always been an indispensable tool for various medical and industrial applications. Medical endoscopy has evolved into various types (e.g., gastroscopy, colonoscopy, bronchoscopy, rhinoscopy, otoscopy, and capsule) depending on specific diagnoses and treatments [1]-[5]. Industrial endoscopes are extensively employed in non-destructive visual inspections of various parts in the aircraft, automobile, and plant industry, including engines, pipes, and turbines [6]. Other applications can be also found in security, rescue, and police/military operations. Recently, low-cost endoscopes are being widely deployed. In particular, a wireless endoscope has become increasingly more popular due to its convenience in use and the significant reduction in its cost [7]-[11]. Most commercial wireless endoscopes have a limited

This work was supported by the Research Program funded by the SeoulTech (Seoul National University of Science \& Technology), Korea.

D. Shim is with SeoulTech (Seoul National University of Science \& Technology), Seoul, Korea (e-mail: dongha@seoultech.ac.kr).

J. Yi is with Haesung Optics Co., Ltd., Suwon, Korea (e-mail: jsyi@hso.co.kr).
FOV (Field of View) of $60^{\circ}$ or less, and they do not have a bending distal tip because of cost limitations. The proposed endoscope employs an ultra-wide-angle lens module to resolve the issue. All lenses in the lens module are injection-molded to reduce the manufacturing costs.

Most low-cost commercial wireless endoscopes employ a parallel CMOS image sensor and a Wi-Fi communication link [7]-[11]. Few of them support the resolution levels above VGA (640x480 pixels), as their configurations have some limitations in supporting a higher video resolution. A parallel sensor with a higher resolution requires interconnections that are more complicated and thereby increases the size of the camera head. In addition, the larger number of connections is responsible for its relatively large power consumption, which is a crucial weakness for battery-powered mobile applications. The Wi-Fi communication link often cannot provide the required data transfer rate for a higher video resolution (above VGA) due to its limited bandwidth. Therefore, commercial Wi-Fi endoscopes often experience a noticeable delay similar to that faced by Wi-Fi cameras due to their low data transfer rates [12]. A new architecture for a wireless endoscope needs to be developed to support higher quality video with a high-speed data transmission capability.

In commercial wireless endoscopes, a camera controller is usually packaged in a camera head along with a parallel image sensor to keep the interconnections short. The camera controller processes the image data and communicates to a terminal device using a generic protocol (e.g., USB). The size and complexity of the camera head can be significantly reduced if it included only the image sensor physically separated from the camera processor. The proposed endoscope employs a low-cost commercial serial image sensor with a MIPI (Mobile Industry Processor Interface) CSI-2 (Camera Serial Interface-2) interface [13]. A flexible cable placed between the image sensor and the camera processor achieves the BCC (Backend-Camera-Controller) architecture [14]. A serial image sensor consumes less power than a parallel one, thereby extending the battery-operation-time of a wireless endoscope. The proposed endoscope employs a high-speed UWB (Ultra-Wide-Band) for the communication link. The wide bandwidth of the UWB link allows transmissions of high-resolution video at a high data transfer rate. The wireless endoscope also supports a Wi-Fi link to take advantage of its flexible interoperability with various mobile devices. A prototype of a high-performance wireless endoscope is implemented using the BCC architecture and the high-speed UWB link. Measurements evaluate the optical, video and latency characteristics of the prototype endoscope. 


\section{ARCHITECTURE OF WIRELESS ENDOSCOPE}

For obvious reasons, a long cable should be avoided while interconnecting a parallel image sensor and a camera processor. First, a long interconnecting cable would introduce a synchronization issue among parallel data and clock channels [15]. Second, multiple parallel data lanes required by the image sensor would make the cable between a parallel image sensor and a camera processor bulky and stiff, limiting the accessibility and maneuverability of the endoscope [15]. Third, the increased complexity of the camera head and the long interconnecting cable would result in additional manufacturing costs. A parallel image sensor with a higher resolution needs more interconnections, which is a major bottleneck in achieving a high resolution endoscope.

The above issues can be resolved by using an image sensor with a serial interface using less interconnection for data lanes. The MIPI CSI-2 standard features a serial, fast, and low-power interface between a peripheral image module and a mobile device, ensuring relatively small dimensions [13].

Fig. 1 shows the structure of the proposed wireless endoscope with the BCC architecture using the MIPI CSI-2 image sensor. The endoscope consists of the camera head, a cable, a camera controller, and a wireless handle. The image sensor in the camera head connects to the camera controller through a multi-strand flexible cable. The camera controller converts the MIPI-enabled serialized digital sensor output into a UVC (USB Video Class) format, and directly communicates the UVC digital video to the wireless handle via a USB 2.0 cable. The wireless handle transmits the video data to a terminal device by either the UWB or the Wi-Fi communication link depending on the selected mode.

Fig. 2 shows the photo of the implemented wireless endoscope prototype. A wireless endoscope with a higher resolution can be achieved using the proposed BCC architecture along with the high-speed UWB link.

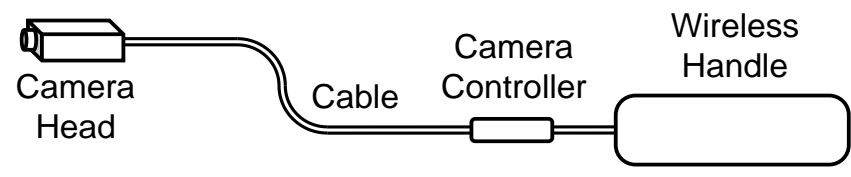

Fig. 1. Configuration of the ultra-wide-angle wireless endoscope with the backend-camera-controller configuration.

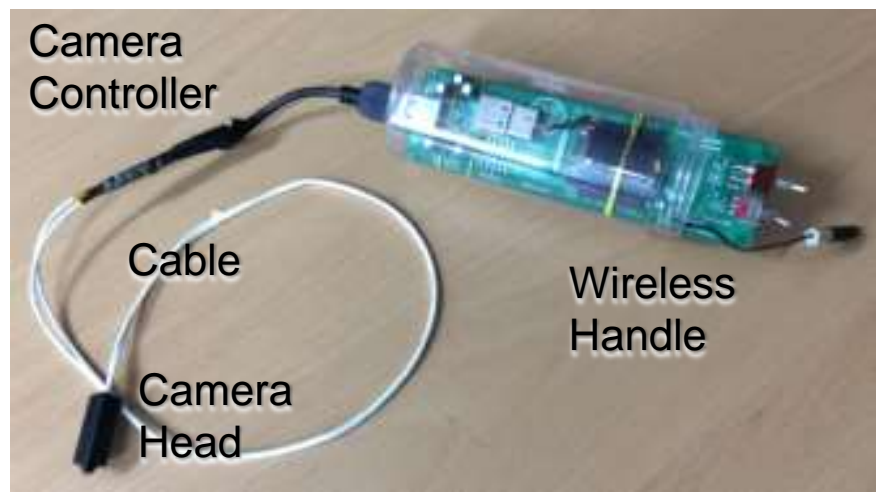

Fig. 2. Photo of the proposed wireless endoscope prototype.

\section{IMPLEMENTATION OF WIRELESS ENDOSCOPE}

\section{A. Camera Head}

Fig. 3 shows a 3-D exploded view of the camera head. It consists of a lens module, a holder, an image sensor, an interface board, and a case. Four aspheric lenses implement the ultra-wide-angle lens module with a $150^{\circ} \mathrm{AOV}$ (Angle of View) [16]. To reduce the manufacturing costs, all lenses are injection-molded using PMMA (Polymethyl Methacrylate) and no glass lenses are used. The AOV of the lens module is estimated to be $\sim 150^{\circ}$ with an F-number of 3.5, and an EFL (Effective Focal Length) of $1.02 \mathrm{~mm}$. The lens module has the diameter and length of 3.5 and $3.1 \mathrm{~mm}$, respectively [16].

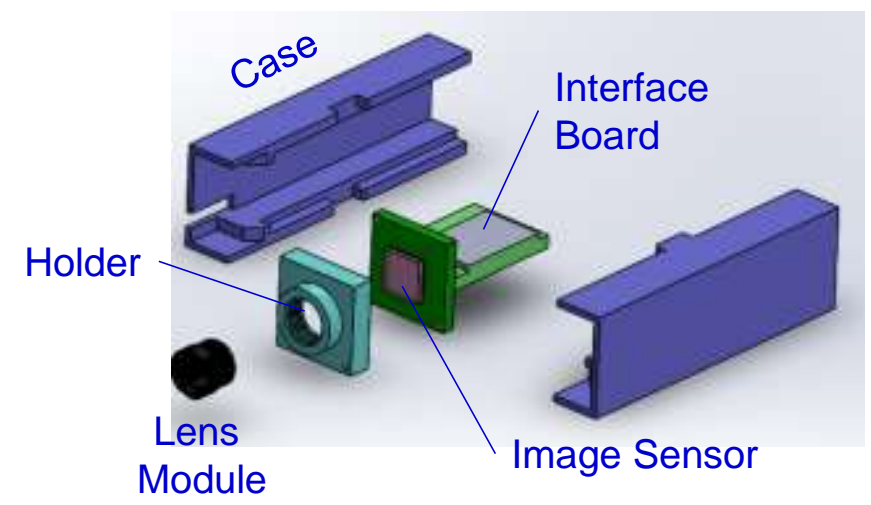

Fig. 3. 3-D exploded view of the proposed camera head.

A commercial CMOS color image sensor reduces the size and cost of the camera module [17]. The image sensor uses the CSI-2 interface of the MIPI standard, which is widely adopted by conventional mobile devices. The MIPI standard features high performance to support a high data rate at low power consumption [13]. The image sensor has a one-lane high-speed CSI-2 serial data interface that reduces the number of interconnections significantly compared to those of a parallel image sensor [17]. It supports the high-definition resolution of $1280 \times 720$ pixels (720p) at a frame rate of $30 \mathrm{fps}$. The package size of the image sensor is $3.8 \times 3.3 \mathrm{~mm}$ with a pixel size of $1.75 \times 1.75 \mu \mathrm{m}$. Its low-power characteristic is especially critical for a mobile device powered by a battery. A parallel image sensor with similar specifications consumes $3 x$ higher power [18]. The image sensor is mounted on a 7x7 mm PCB soldered perpendicular to the interface board. The interface board has a compact size of $7 \times 11 \mathrm{~mm}$, and does not include any other electronic component. The interface board connects to the camera controller through a $\sim 70-\mathrm{cm}$ long flexible cable containing multiple strands. The 14-strand cable offers interconnections for the MIPI data/clock lanes, the serial camera control bus, the system clock, the power supplies, and grounds. Although the cable has 24 strands, only 14 are used as the cable was chosen based on easy availability.

The camera unit is packaged within a plastic case. Fig. 4 shows a photo of the packaged camera head. The size of the camera module is $8 \times 8 \times 26 \mathrm{~mm}$. The inset in the figure shows an illumination unit mounted on the holder. The unit has two white small-footprint chip LEDs with a size of $1.6 \times 0.8 \mathrm{~mm}$ each. The camera head can be further reduced using a smaller image sensor with more compact design [16]. 


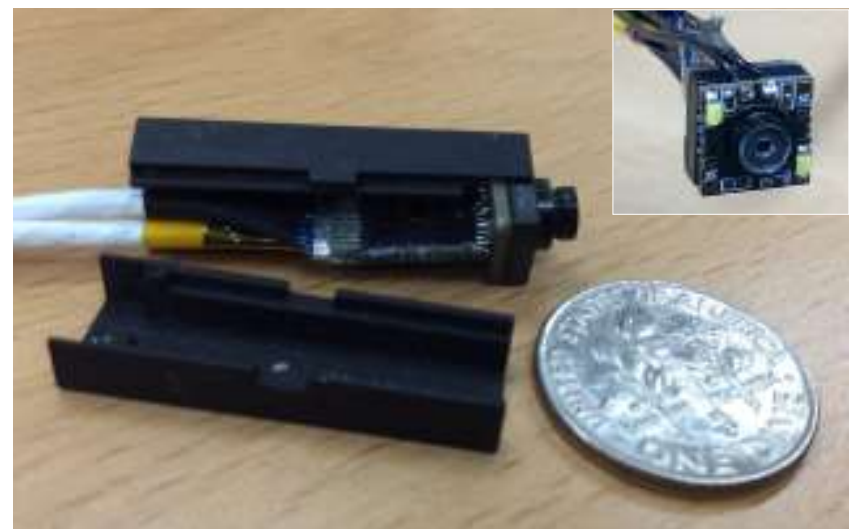

Fig. 4. Photo of the manufactured camera head. The inset show the illumination unit mounted on the holder.

\section{B. Camera Controller}

Fig. 5 shows the top and the bottom side of the camera controller. A camera processor processes the video data and communicates with the wireless handle via the UVC interface [19]. The unit can transfer videos up to FHD (1920x1080 pixels) at $30 \mathrm{fps}$, streaming in the uncompressed YUY2 or the MPEG format. A serial flash memory is mounted for initialization of the image sensor [20]. The size of the camera controller board is $7 \times 55 \mathrm{~mm}$. A $~ 70-\mathrm{cm}$ long multi-strand cable connects the camera head and the camera controller. The output of the camera controller connects to the wireless handle via a $\sim 10$-cm long USB 2.0 cable, although the camera controller can be readily integrated within the wireless handle if necessary.

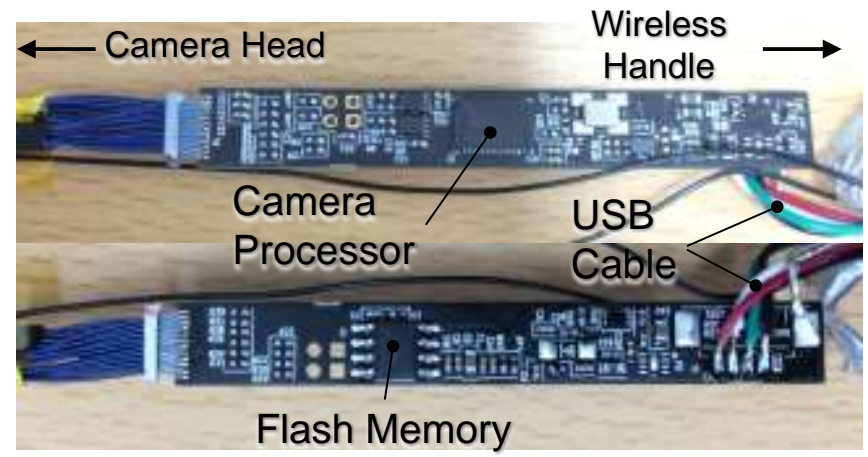

Fig. 5. Top and bottom side of the camera controller for the wireless endoscope.

\section{Wireless Handle}

Fig. 6 shows the configuration of the wireless handle. It transmits video data from the camera controller wirelessly using the UWB or the Wi-Fi link. The UWB or the Wi-Fi unit is exclusively powered (selected) through the mode switch. The USB cable from the camera controller connects to the input of the USB diplexer that delivers the video signal to either the UWB or the Wi-Fi unit depending on the position of the mode switch. The regulator converts the $5 \mathrm{~V}$ from the booster/charger to $3.3 \mathrm{~V}$ to supply the USB diplexer. Since the USB diplexer operates under a different supply voltage, the level shifter translates the control voltage level from the mode switch. The booster/charger converts the output voltage of the battery and charges it from an external source.

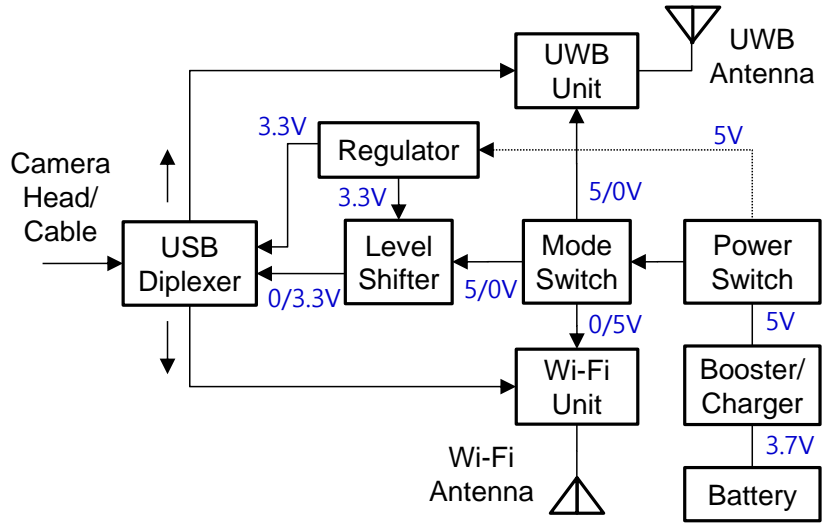

Fig. 6. Configuration of the wireless handle.

UWB is a communications technology with a wide bandwidth (typically defined as greater than $20 \%$ of the centre frequency or $500 \mathrm{MHz}$ ) [21]. It is typically characterized by high-speed, ultra-low power, and short range. UWB radios operate with very low transmit power and do not cause interference with electronic devices or other types of radios.

A commercial WUSB (Wireless USB) adaptor module implements the high-speed UWB unit [22], [23]. The UWB unit includes a broadband biconical antenna, widely adopted in UWB applications [24]-[26]. The size of the unit is $17 \times 34 \times 4$ $\mathrm{mm}$. There is a report of a wireless camera using the UWB unit [27]. The camera transmits videos with a resolution of $2048 \times 1536$ pixels (QXGA) at a frame rate of $15 \mathrm{fps}$ in an MJPEG (Motion JPEG) format [27]. The maximum data transfer rate reached was $\sim 40 \mathrm{Mbps}$. Therefore, this wireless handle should be able to support a camera head with a resolution higher than 1280x720 pixels.

A Wi-Fi UVC video router is employed for the Wi-Fi unit [28]. It is compliant with the IEEE $802.11 \mathrm{~b} / \mathrm{g} / \mathrm{n}$ standards. The unit works as an AP (Access Point) and any mobile device can directly connect to it without going through a Wi-Fi network. The size of the module is $35 \times 25 \times 18 \mathrm{~mm}$, and this includes the PIFA (Planar Inverted-F Antenna) at the edge of the unit.

The USB signal from the camera controller connects to the UWB or the Wi-Fi unit depending on the selected communication mode. A high-bandwidth electronic USB diplexer handles the switching. It has a maximum on resistance of $10 \Omega$ with a variation of $0.35 \Omega$ [29]. It is powered by $3.3 \mathrm{~V}$ from a regulator, as its supply voltage range $(3.0-4.3 \mathrm{~V})$ is lower than $5 \mathrm{~V}$.

The regulator converts the $5 \mathrm{~V}$ from the booster/charger to 3.3 $\mathrm{V}$ to power the USB diplexer and the level shifter, and is implemented using an LDO (Low Dropout) regulator featuring low noise and low power characteristics [30].

Since the maximum output voltage of the mode switch $(5 \mathrm{~V})$ exceeds the maximum input control voltage level of the USB diplexer (3.3 V), a level shifter is employed to make the voltage levels compatible and is implemented using a Schmitt-trigger inverter [31]. The level shifter is powered by the $3.3 \mathrm{~V}$ generated by the regulator.

The mode switch, connected to the power switch, selectively powers the UWB or the Wi-Fi unit. It also provides the control signal for the USB diplexer through the level shifter. The switch is a mechanical toggle unit with a DPDT (Double Pole Double Throw) configuration. 
The power switch is a mechanical toggle unit with an SPDT (Single Pole Double Throw) configuration and is placed between the booster/charger and the mode switch to control the supply power to the wireless endoscope.

Over time, a slight degradation in the data transfer rate was observed caused by the heat from the communication units. A heat sink (an aluminium cooling fin and a ceramic plate) is mounted on each unit to mitigate the temperature rise, and is shown in Fig. 7. The battery is a cylindrical lithium-ion type generating $3.7 \mathrm{~V}$, and a capacity of $2600 \mathrm{mAh}$ [32].

The booster/charger steps up the battery output voltage of 3.7 $\mathrm{V}$ to $5.0 \mathrm{~V}$ to supply the UWB/Wi-Fi module, the regulator, the camera controller, and the camera head. The unit also charges the battery from an external $5 \mathrm{~V}$ source. All components are mounted on a two-layer FR-4 PCB with a size of 140x40 mm. The top and the bottom sides of the wireless handle are shown in Fig. 7.

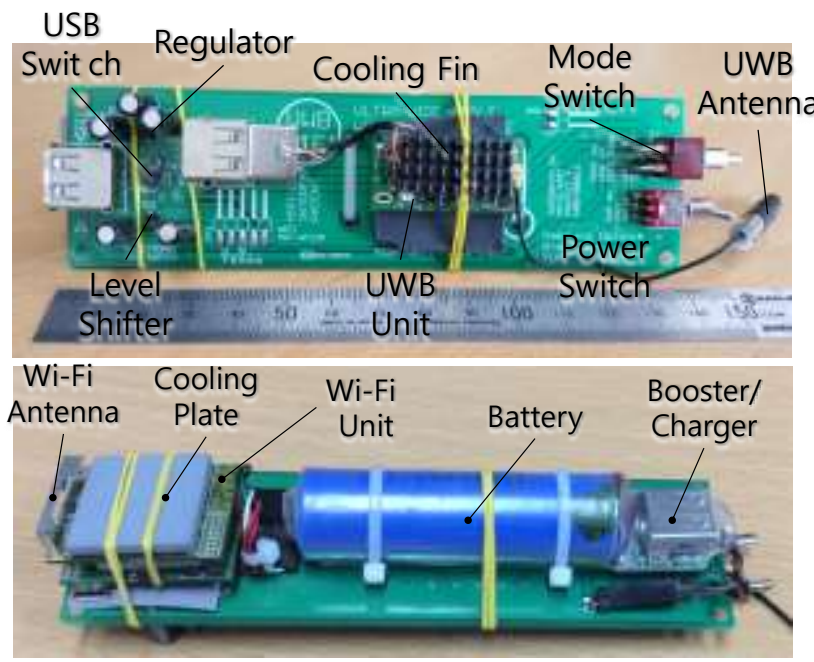

Fig. 7. Top and bottom side of the wireless handle.

\section{MEASUREMENT RESUltS}

\section{A. Field of View}

The FOV measurement on the proposed endoscope is compared with that on a commercial unit (WF200) [11]. Two camera heads are attached and positioned at the same height above a grid pad (Fig. 8). The vertical distance between the camera heads and the pad is $38 \mathrm{~mm}$. The size of a square cell in the pad is $10 \times 10 \mathrm{~mm}$. The transmitted video from the proposed and the commercial unit is displayed via a laptop and a mobile tablet, respectively [33]. The horizontal and the vertical FOV of the proposed endoscope is estimated to be $69^{\circ}$ and $117^{\circ}$, respectively, with the diagonal FOV as $130^{\circ}$ (Fig. 9). Moderate barrel distortion is observed due to the ultra-wide-angle characteristic of the lens module. To the best of the author's knowledge, the proposed endoscope has the largest FOV among all existing wireless endoscopes. The measured FOV is smaller than the AOV of the lens module $\left(150^{\circ}\right)$ since the image sensor can cover only a part of an incident image through the lens module. The horizontal, the vertical, and the diagonal FOV of the commercial unit are estimated to be $46^{\circ}, 33^{\circ}$, and $55^{\circ}$, respectively (Fig. 10). The FOV of the proposed endoscope is more than twice that of the commercial unit.

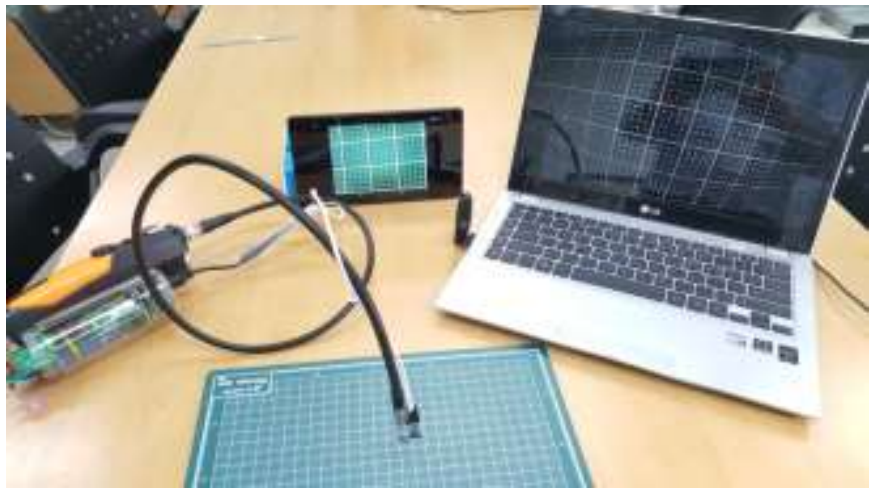

Fig. 8. FOV measurement setup.

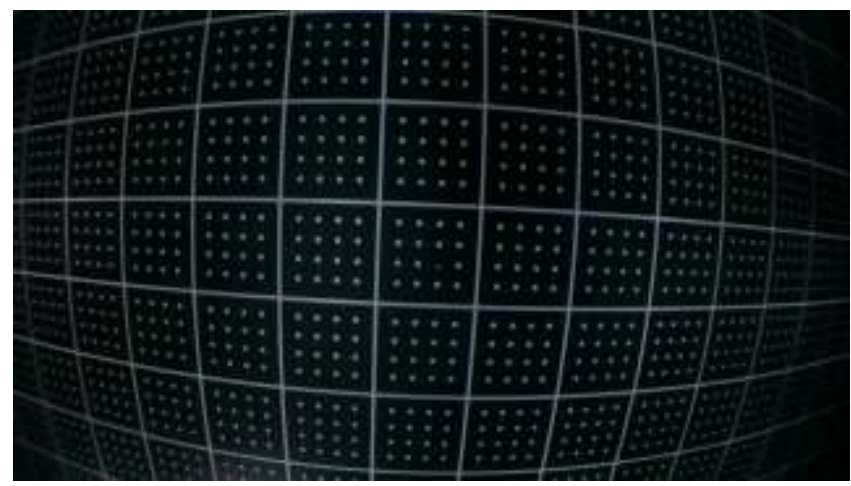

Fig. 9. Grid photo by the proposed ultra-wide-angle endoscope.
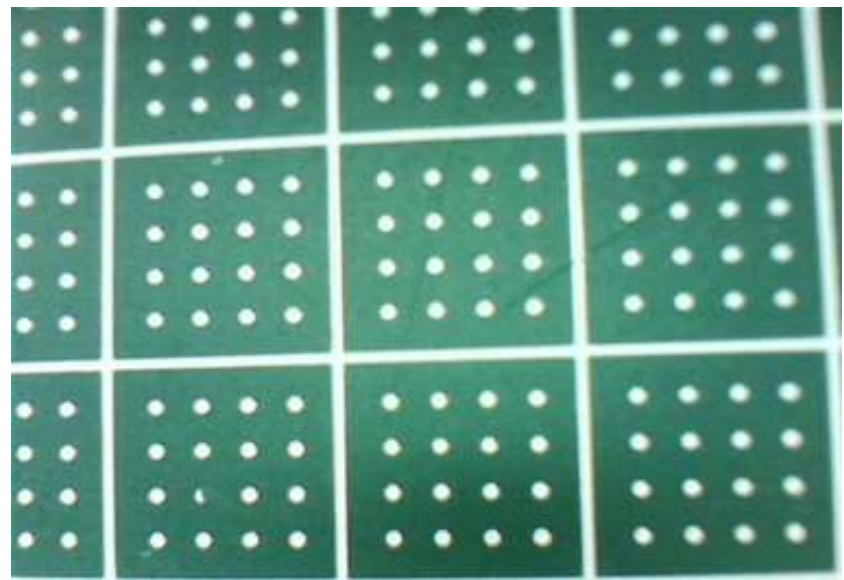

Fig. 10. Grid photo by the commercial wireless endoscope (WF200) [11]

\section{B. Video Performance}

The video performance of the wireless endoscope is evaluated along with the resolution, the frame rate, and the data transfer rate. The wireless handle and a receiver (UWB or Wi-Fi adaptor) are placed within LOS (Line-of-Sight) at a distance of $2 \mathrm{~m}$. A free and open source cross-platform multimedia player and a framework (VLC) are used to measure the video performance [34]. The endoscope operates at $720 \mathrm{p}$ HD resolution (1280x720 pixels) at a frame rate of $30 \mathrm{fps}$ in the UWB mode. The maximum data transfer rate reaches $\sim 37$ Mbps. The endoscope operates in the Wi-Fi mode at the same resolution, but has a much lower data transfer rate ( $~ 8 \mathrm{Mbps}$ Max.). Although VLC does not provide a frame rate in the Wi-Fi mode, after comparing the measured data transfer rates, it is estimated to be more than four times lower than the frame rate of the UWB mode. 


\section{Latency}

The video latency of the UWB link is measured using the setup shown in Fig. 11 [27]. The camera head is positioned to observe a running stopwatch. Its video output is transmitted to the host UWB adapter plugged into a laptop and is displayed on the screen. The video latency can be estimated from the time difference of the original and the captured stopwatch window. The camera has a latency of $\sim 0.1 \mathrm{sec}$ for the UWB mode. To measure the latency in the Wi-Fi mode, an embedded adaptor in the laptop is connected to the Wi-Fi unit in the wireless handle. Following the same method, the Wi-Fi unit shows a latency of $\sim 0.5 \mathrm{sec}$ (Fig. 12). The larger latency in the Wi-Fi mode of operation can be explained mainly by the lower data transfer rate and the lower frame rate. The overall power consumption of the endoscope is measured to be 1.5 and $2.1 \mathrm{~W}$ at the UWB and the Wi-Fi operation modes, respectively. The performances of various commercial endoscopes are compared in Table I.

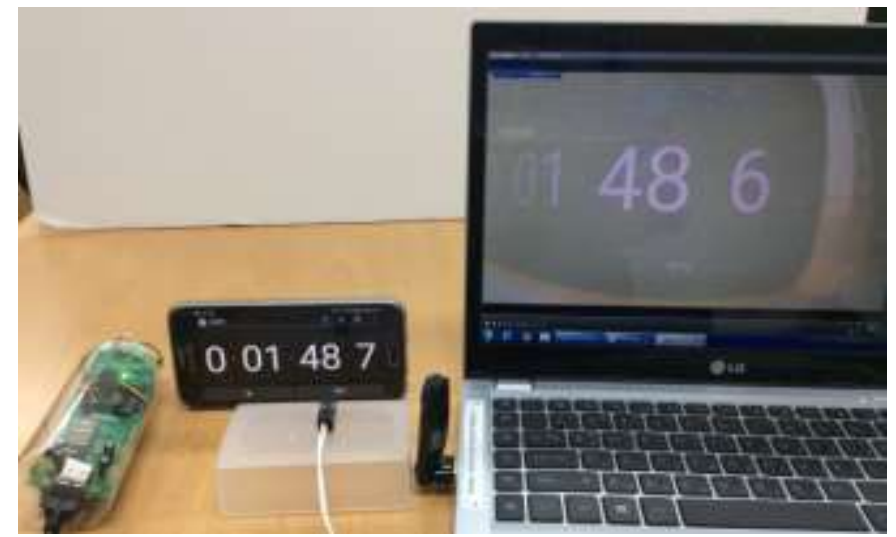

Fig. 11. Latency measurement in UWB communication mode.

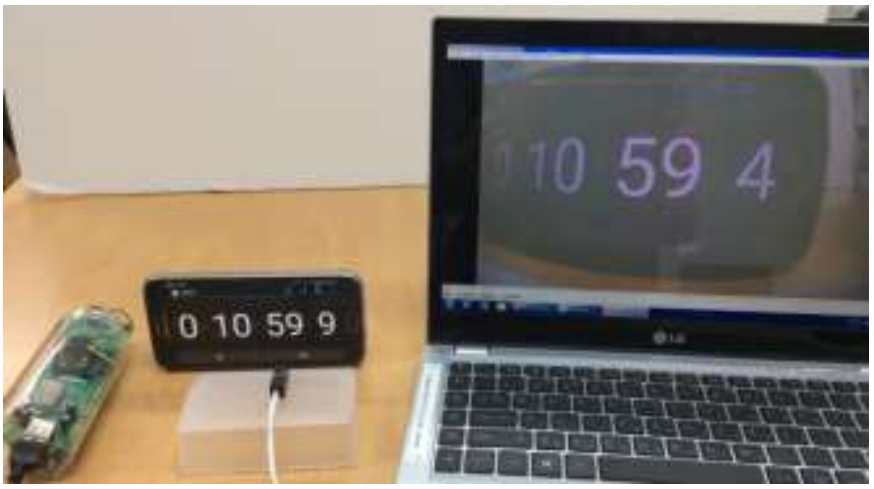

Fig. 12. Latency measurement in Wi-Fi communication mode

\section{CONCLUSION}

An ultra-wide-angle wireless endoscope was demonstrated with an $\mathrm{FOV}$ of $130^{\circ}$ and with $\mathrm{HD}$ resolution $(1280 \times 720$ pixels). The lens module is implemented with all-plastic lenses to have an $\mathrm{AOV}$ of $150^{\circ}$. The lenses are manufactured by a plastic injection-molding process to reduce the cost. The endoscope employs a BCC architecture to physically separate the camera controller from the camera head. The compact structure of the camera head is achieved using a serial CMOS image sensor with a MIPI CSI-2 interface. The horizontal and the vertical FOV of the proposed endoscope are estimated to be $69^{\circ}$ and $117^{\circ}$, respectively, with the diagonal FOV as $130^{\circ}$.
TABLE I

SUMMARY OF PERFORMANCE COMPARISON

\begin{tabular}{cccccc}
\hline \hline Ref. & $\begin{array}{c}\text { FoV } \\
\text { (deg.) }\end{array}$ & $\begin{array}{c}\text { Resolution } \\
\text { (pixels) }\end{array}$ & $\begin{array}{c}\text { Frame } \\
\text { Rate } \\
\text { (fps })\end{array}$ & $\begin{array}{c}\text { Dia. } \\
(\mathrm{mm})\end{array}$ & $\begin{array}{c}\text { Commu- } \\
\text { nication }\end{array}$ \\
\hline$[7]$ & 60 & $640 \times 480$ & 30 & 9 & $\mathrm{WiFi}$ \\
{$[8]$} & $\mathrm{NA}$ & $640 \times 480$ & $\mathrm{NA}$ & 9.8 & $\mathrm{WiFi}$ \\
{$[9]$} & 50 & $640 \times 480$ & 30 & 17 & $\mathrm{WiFi}$ \\
{$[10]$} & 105 & $1.3 \mathrm{M}$ & $\mathrm{NA}$ & $\mathrm{NA}$ & $5.8 \mathrm{GHz}$ \\
{$[11]$} & 60 & $1280 \times 720$ & $\mathrm{NA}$ & 8.5 & $\mathrm{WiFi}$ \\
This work & 150 & $1280 \times 720$ & $30 /$ & 11.2 & $\mathrm{UWB} /$ \\
\end{tabular}

The wireless handle includes the UWB and the Wi-Fi communication units for the wireless video transmission. The UWB link supports a maximum data transfer rate of $\sim 37 \mathrm{Mbps}$ to transmit videos with a resolution of $1280 \times 720$ pixels and a frame rate of $30 \mathrm{fps}$ in the MJPEG format. The endoscope also supports the Wi-Fi operation mode for enhancing the interoperability with various mobile devices, although at a relatively lower speed ( $\sim 8 \mathrm{Mbps}$ Max.). The latency of the endoscope is measure to be $\sim 0.1 \mathrm{sec}$ in the UWB mode. A noticeable latency $(\sim 0.5 \mathrm{sec})$ is observed in the $\mathrm{Wi}-\mathrm{Fi}$ mode due to its lower data transfer rate.

These results suggest that the proposed BCC architecture along with the high-speed UWB link should be able to keep pushing the resolution of a wireless endoscope above HD-levels while keeping the size of the camera head and the cable compact. The proposed endoscopy structure will open up the path to a higher-performance yet low-cost wireless endoscopy.

\section{REFERENCES}

[1] J. M. Edmonson, "History of the instruments for gastrointestinal endoscopy," Gastrointestinal Endoscopy, vol. 37, no. 2, pp. S27-S56, 1991. DOI: http://dx.doi.org/10.1016/S0016-5107(91)70910-3

[2] S. J. Spaner and G. L. Warnock, "A Brief History of Endoscopy, Laparoscopy, and Laparoscopic Surgery," J. Laparoendoscopic \& Advanced Surgical Techniques, vol. 7, no. 6, pp. 369-373, Jan. 2009.

[3] A. R. Haas, A. Vachani and D. H. Sterman, "Advances in Diagnostic Bronchoscopy," Amer. J. Respiratory and Critical Care Medicine, vol. 182, no. 5, pp. 589-597, 2010. DOI: $10.1164 / \mathrm{rccm} .201002-0186 \mathrm{CI}$

[4] C. A. Rawlings, "Diagnostic Rigid Endoscopy: Otoscopy, Rhinoscopy, and Cystoscopy," J. Amer. Animal Hospital Association, vol. 39, pp. 849-868, 2009.

[5] T. Nakamura and A. Terano, "Capsule endoscopy: past, present, and future," J. Gastroenterology, vol. 43, no. 2, pp. 93-99, 2008

[6] "The World of Industrial Endoscopy", Karl Storz GmbH [online]. https://www.karlstorz.com/cps/rde/xbcr/karlstorz_assets/ASSETS/34330 42.pdf.

[7] Flex-WiFi-9mm, Vividia [online]. http://www.amazon.com/VividiaWaterproof-Inspection-Borescope-Endoscope/dp/B00DCVZQGC/ref=sr _1_3?ie=UTF8\&qid=1390623081\&sr=8-3\&keywords=wifi+endoscope.

[8] DCiS1, General Tool [online]. http://www.generaltools.com/ iborescope.html.

[9] BR200, Extech Instruments [online]. http://www.extech.com.

[10] MD-910, Dongguan Magenta Medical Instrument Co. [online]. http://www.dgmagenta.com.

[11] WF200, CrazyFire [online]. http://www.amazon.com/CrazyFire\% C2\%AE-WF200-Endoscope-Inspection-Flashlight/dp/B00H2JCENS

[12] R. Hill, C. Madden, A. van den Hengel, A., H. Detmold and A. Dick, "Measuring Latency for Video Surveillance Systems," Digital Image Computing: Techniques and Applications (DICTA), Dec. 2009, pp. 89-95. doi: 10.1109/DICTA.2009.23 
[13] MIPI camera interface specifications [online]. http://mipi.org/specifications/camera-interface.

[14] E. Baum and P. Schwarz, "Endoscopic instrument," U.S. Patent 8690759 B2, 2011.

[15] J. R. Freer, "Serial versus parallel," in Computer Communications \& Networks, Taylor \& Francis: London and New York, 1996, pp. 8-9.

[16] D. Shim, J. Yeon, J. Yi, J. Park, S. N. Park and N. Lee, "A Wide-angle Camera Module for Disposable Endoscopy," Optical Review, vol. 23, no. 4, pp. 596-600, Aug. 2016

[17] OV9728 datasheet, OmniVision Technologies Inc. [online]. http://www.ovt.com/download_document.php?type=sensor\&sensorid=1 31.

[18] OV10633 datasheet, OmniVision Technologies Inc. [online]. http://www.ovt.com/download_document.php?type=sensor\&sensorid=1 10.

[19] SPCA2088A, Sunplus Innovative Technology Inc. [online]. http://www.sunplusit.com/english/products/pccamera/SPCA2088A.aspx

[20] PM25LV512 / PM25LV010 datasheet, Programmable Microelectronics Corp. [online]. http://www.alldatasheet.com/datasheet-pdf/pdf/153937 /PMC/PM25LV512-25SCE.html

[21] K. Siwiak and D. McKeown, Ultra-Wideband Radio Technology. Wiley, 2004.
[22] AL5616 (UWB Radio Card Adapter), Alereon [online]. http://www.alereon.com/products/reference-design-boards/al5616-world wide-wireless-usb-adapter.

[23] Wireless USB (WUSB) [online]. http://www.usb.org/developers/wusb/.

[24] S. Ida and D. Muto, "Biconical antenna," US7221326 B2, 2007.

[25] P. S. Carter, "Short Wave Antenna," U.S. Patent 2175252, 1939.

[26] O. Lodge, "Electric Telegraphy," U.S. Patent 609154, 1898.

[27] D. Shim, H. Kim, M. Lee, J. Kim, J. Yi, K. Kang, J. Kang and N. Lee, “An Ultra-Wide-Angle Wireless Camera using Ultra Wide Band Communication,” Int. J. Sensor Networks, vol. 19, no. 1, pp. 37-45, 2015.

[28] MST-WF12M datasheet, Misumi Electronic Corp. [online]. http://www.misumi.com.tw/pdf/2013/MST_WF12m.pdf.

[29] TS3USB30 datasheet, Texas Instruments [online]. http://www.ti.com/product/TS3USB30.

[30] LT1761 datasheet, Linear Technology [online]. www.linear.com/docs/2732.

[31] MC74VHC1GT14 datasheet, On Semiconductor [online]. http://www.onsemi.com/pub_link/Collateral/MC74VHC1GT14-D.PDF.

[32] ICR16850-26H, SDI [online]. http://www.samsungsdi.co.kr/ lithium-ion-battery/it-devices/power-bank.html.

[33] Nexus 7, Google [online]. https://store.google.com/product/nexus_7.

[34] VLC Media player, Video LAN [online]. http://www.videolan.org/vlc/index.ko.html. 